\title{
Synchronous Esophageal and Renal Malignancies with Unexpected Site of Metastasis: A Case Report
}

Sharhanin Bahrudin

Kulliyyah of Medicine, International Islamic University Malaysia, Kuantan Pahang.

Introduction: Esophageal cancer is the fifth most common cancer in Asia. Synchronous malignancies with an esophageal malignancies is not uncommon however synchronous esophageal malignancy and renal cell carcinoma is rare. Case Report: A 70 years old non smoker man with comorbid of hypertension and diabetes mellitus presented with multiple episode of hemoptysis and pleuritic chest pain within one day duration. He also complaint of worsening dysphagia to solid food for 1 month duration with constitutional symptoms. Otherwise he does not have any other symptoms. Clinically he was pale and cachexic, otherwise all systemic examination was unremarkable. His blood investigation reveal low hemoglobin level of $9.9 \mathrm{~g} / \mathrm{L}$ with normal coagulation and renal profile. He underwent Oesophagoduodenoscopy which showed a tumor narrowing the lumen of the lower esophagus. Biopsy of the tumor revealed esophageal adenocarcinoma. CT thorax, abdomen and pelvis showed smooth circumferential wall thickening of the distal part of the esophagus near the gastroesophageal junction. Incidentally, there were heterogenous enhancing lobulated mass seen at the lower pole of left kidney with no obstructive uropathy features. Otherwise, others solid organ were normal. A left renal mass biopsy performed revealed renal cell carcinoma. An oncologist opinion was gathered for initiation of palliative chemotherapy however he developed symptomatic malignant pleural effusion which need a drainage. HRCT Thorax revealed multiple bilateral intrapulmonary nodule. He also complaint of a new right upper eyelid mass that increasing in size with contact bleeding however does not affect his visual. An assessment from an ophthalmologist and CT orbital performed consistent with features of a metastasis. Conclusion: Synchronous malignancies with an esophageal malignancies is not uncommon. Most common site reported is head and neck followed by lung malignancies. However synchronous esophageal malignancies and renal cell carcinoma is rare. Esophageal malignancies often have distant metastasis to the liver, lung and bone however rare to the eyelid. 